\title{
Open doors for research in Japan
}

Thomas W. Ebbesen

Many Japanese companies are keen to recruit foreign scientists, and as employers they have much to offer. More researchers should take up the opportunities available for work in Japan.

THE difficult research conditions in many of Japan's universities have been discussed in recent Commentaries ${ }^{1.2}$; no doubt such circumstances discourage foreign scientists from seeking employment in Japan's academic institutions. But more than 80 per cent of Japan's research and development is carried out in the private sector where, because of the phenomenal growth of Japanese industry, the $R \& D$ environment is evolving rapidly in terms of funding, direction and hiring practices. There are challenging opportunities here for foreign researchers.

Just as public institutions in Japan now have many postdoctoral fellowships available, many companies have also opened their doors to foreign scientists. A survey conducted by the Tokyo office of the US National Science Foundation in 1985 showed that more than 60 per cent of the responding companies were either ready, or would be ready, to employ foreign researchers in their laboratories ${ }^{3}$. Although it remains to be seen whether these plans have been translated into action, it is clear that many companies now have foreigners on their staff. Companies believe that recruitment of foreigners supports the trend towards increased basic research by providing fresh input from people with different perspectives and backgrounds.

In general, the number of foreigners working in the private sector, both academic and industrial, is much greater than in the public sector. In 1987, for instance, there were 392 foreign full professors in private universities and 12 in national and public institutions. The reason for this large difference is probably that public institutions have not provided a general mechanism for tenure of their foreign employees ${ }^{2}$. As a result, most of them wishing to stay in Japan end up with a job in a private university or in industry. Among the more progressive companies, both temporary and permanent employment are provided. In 1988 , for instance, among the 522 foreign graduates of Japanese universities who were hired by Japanese companies, more than 40 per cent had permanent posts ${ }^{5}$. This large percentage is very encouraging as the offer of permanent employment to these foreigners who have little trouble with Japanese suggests openness and fair treatment irrespective of nationality.

Like the public sector ${ }^{2}$, the private sector rarely advertises jobs in journals. Traditionally, recruiting is done through direct contact, a human network, between university professors, company employees and the prospective candidates. In general, recruiting is not as competitive as it is, for instance, in the United States. Japanese companies rely heavily on their reputation and desirability to attract the best graduates. Foreign scientists are also recruited through direct contacts or by recommendation by a third party. Human relations being of the utmost importance to the management, evaluation of the person both as a 'social being' and as a scientist has a high priority.

Foreign researchers are generally hired on yearly renewable contracts such as postdoctoral positions. Even in the companies that offer permanent employment, there is generally a period of adaptation and mutual evaluation before an employee achieves permanent status. Permanent employment requires strong longterm commitment by both parties.

There is a general belief that Japanese institutions have a much more rigid hierarchy than those in other countries. But this depends on the type of institution. Company laboratories can be quite democratic: the difference in salary between the lowest and highest ranks is small, and regular meetings are held at all levels to exchange information and discuss policies. Managements have their systems of checks and balances. For instance, a lower-ranking researcher can request a transfer to another group if he or she is dissatisfied with working conditions. So it is important to managers to have a good relationship with their subordinates and colleagues, and decisions are generally taken by consensus and compromise. Although some complain that such a system does not allow for clear-cut and quick decisions, it does mean that human relations are smooth.

Because of this system, the period between a potential employee's first contact with the company and his or her hiring can be very long. It takes at least 5 to 6 months to process an application and for visa formalities. Furthermore, foreign candidates unfamiliar with Japanese practices often find it difficult to judge the true level of commitment from their correspondence with a company. Although knowledge of Japanese is generally not essential for postdoctoral or visiting status, some knowledge of the Japanese language and culture is very useful and can decrease cross-cultural barriers which are mutually frustrating.

As was pointed out by Yamamoto' ${ }^{1}$, the overall expenditure on research by industry is much larger than that by the public sector (although spending on basic as opposed to applied research remains to be considered). The difference is even greater when high-tech industry is considered: for example the electronics industry obviously depends on innovation and improvement, which in turn requires significant spending on research and development. The average amount per researcher spent on capital equipment in the larger company laboratories can only be matched by the most powerful research groups (koza) of public institutions, and is comparable to that available in the equivalent top Western companies.

With the favourable financial position of many companies in Japan at the moment, research investment is becoming more stable, long-term and more orientated to basic science. In a few companies, research is being done that is decades ahead of development. Companies offering such opportunities are attractive alternatives to an academic career, generally considered more prestigious by young Japanese researchers. These conditions are also attracting an increasing number of foreigners.

There is a problem which hinders scientific exchange. Young postdoctoral scientists, especially from the United States, are often neither encouraged to go abroad nor credited for their scientific experience there, so postdoctoral positions in Japan allocated to US researchers are often undersubscribed despite the efforts of agencies like the US National Science Foundation and the Science and Technology Agency of Japan ${ }^{2}$. It is hard to understand the reasons for this attitude as foreign companies are building $R \& D$ facilities in Japan to pool the local resources, and hire Japanese scientists from rival companies. Complaints about the closed nature of Japan's research are no longer appropriate; rather it is time to encourage researchers to take advantage of the many opportunities now on offer in Japan's private and public sectors.

Thomas W. Ebbesen is in the Fundamental Research Laboratories of NEC Corporation, Tsukuba 305, Japan.

1. Yamamoto, A. Nature 339, 575-576 (1989)

2. Siddiqui, S.S. Nature 340, 337-338 (1989)

3. National Science Foundation Tokyo Office Report Memorandum No. 86 (1985)

4. Gakko Kihon Chosa Hokokusho (Ministry of Education, 1987).

5. Ministry of Justice Report on Foreign Student Employment (in Japanese) (1989). 